\title{
Reoperation for herniated thoracic discs
}

\section{Curtis A. Dickman, M.D., Daniel Rosenthal, M.D., and John J. Regan, M.D.}

Division of Neurological Surgery, Barrow Neurological Institute, St. Joseph's Hospital and Medical Center, Phoenix, Arizona; Neurochirurgische Praxisgemeinschaft, Bad Homburg, Germany; and Texas Back Institute, Plano, Texas

In this review the authors address the surgical strategies required to resect residual thoracic disc herniations. Fifteen patients who had undergone prior thoracic discectomy and who harbored residual or incompletely excised symptomatic thoracic discs were reviewed retrospectively. The surgical procedures that had failed to excise the herniated discs completely included 11 posterolateral approaches, one thoracotomy, and three thoracoscopic procedures. Of the incompletely resected or residual disks 13 were central calcified, two were soft, 12 were extradural, and three were intradural discs. Indications for reoperation were often multiple in each patient and included misidentification of the level of disc disease at the initial operation (five cases), abandoning the procedure because of intraoperative spinal cord injury (three cases), inadequate visualization of the pathology (eight cases), migration of a soft disc fragment within the spinal canal (one case), and intradural disc extension (three cases). The symptoms at the time of reoperation included myelopathy in 13 patients and radicular pain in two. The mean interval before reoperation was 150 days (range 1 day-4 years). The reoperation procedures included one thoracotomy and 14 video-assisted thoracoscopic procedures performed ipsilateral (11 cases) or contralateral (four cases) to the site of the initial surgery.

The herniated disc material was excised completely in all 15 cases without causing new neurological deficits. Reoperation complications included atelectasis in three patients, intercostal neuralgia in two, a loosened screw that required removal in one, and a cerebrospinal fluid leak in one patient. Of the 13 patients who experienced myelopathy preoperatively, 10 recovered neurological function and three stabilized. All patients with radicular pain improved.

Calcified, large, broad-based, centrally located, or transdural thoracic disc herniations can be difficult to resect. These lesions require a ventral operative approach to visualize the dura adequately for a safe and complete resection.

Key Words * disc herniation * discectomy * thoracic spine * thoracoscopy

Herniated thoracic discs can present formidable technical and surgical challenges and can be difficult to remove. Discs that are large and broad based, calcified or ossified, those that extend transdurally, and 
those that invaginate the dura and spinal cord are the most challenging lesions to treat surgically. In relatively few reported surgical series is there a discussion of the treatment of herniated thoracic discs.[1-4,6,11,13-15,17-24,26-28] Furthermore, the literature does not address surgical treatment failures or strategies for the reoperation of residual or recurrent thoracic disc herniations.

Working among three tertiary referral centers that specialize in the treatment of thoracic discs, we have had the opportunity to treat 15 patients surgically for symptomatic residual thoracic discs. This experience has allowed us to examine the factors that contributed to incomplete disc removal and to recommend several reoperative strategies to ensure complete removal of these lesions while causing minimal morbidity.

\section{CLINICAL MATERIAL AND METHODS}

The clinical records, radiographs, and imaging studies of 15 patients who had undergone prior thoracic discectomy and who harbored residual, incompletely excised symptomatic thoracic disc herniations were reviewed retrospectively. This number represents $8 \%$ of the 187 patients with thoracic disc disease referred for surgery to our three facilities between 1992 and 1996. Their original radiographs and imaging studies, clinical records, and operative records were reviewed, and the original surgeons were usually contacted to discuss the original operations.

At the time of referral to our facilities for reoperation, the diagnostic studies, clinical characteristics, residual disc material, reoperative strategies, and clinical outcome of the patients were assessed. The details of the reoperative procedures, complications, and postoperative course were examined thoroughly. Postoperative computerized tomography (CT) scans and/or magnetic resonance (MR) images were obtained in all patients. Follow-up review consisted of outpatient office visits in which clinical examinations were performed and radiographs reviewed to assess spinal alignment, as well as evaluation of any imaging studies that were performed. In all patients either postoperative MR imaging or CT studies had been perfomed to assess the extent of decompression. In all patients who underwent spinal reconstruction, internal fixation, and fusion procedures, follow-up plain radiographs were obtained of the thoracic spine, including lateral flexion and extension views. The radiographs were evaluated for the presence of trabecular bone bridging on the fusion surfaces, motion on dynamic radiographs, and instrumentation loosening or breakage. The x-ray films and CT or MR imaging studies were evaluated by the authors and by independent radiologists.

\section{RESULTS}

The surgical procedures first performed to treat thoracic disc herniations included 11 transpedicular or costotransversectomy approaches, one thoracotomy, and three thoracoscopic approaches. In all 15 cases, the initial excision was unsuccessful. The location of the disc herniations are provided in Table 1. 


\section{TABLE 1}

LOCATION OF THORACK DISC HERNIATIONS

IN 15 PATIENTS UNDERGOING REOPERATION

\begin{tabular}{|cc|}
\hline \hline Disc Leve & No. of Patierts \\
\hline T5-6 & 1 \\
$T 6-7$ & 1 \\
$T 7-8$ & 4 \\
$T 8-9$ & 5 \\
$T 9-10$ & 3 \\
$T 10-11$ & 1 \\
\hline
\end{tabular}

There were 13 centrally located calcified or ossified discs and two soft disc herniations (Fig. 1). Twelve of the discs were extradural and three extended transdurally. The symptoms at the time of reoperation included progressive myelopathy due to spinal cord compression (nine cases), persistent myelopathy or myeloradiculopathy without neurological recovery (four cases), and isolated thoracic radicular pain without myelopathy (two cases).
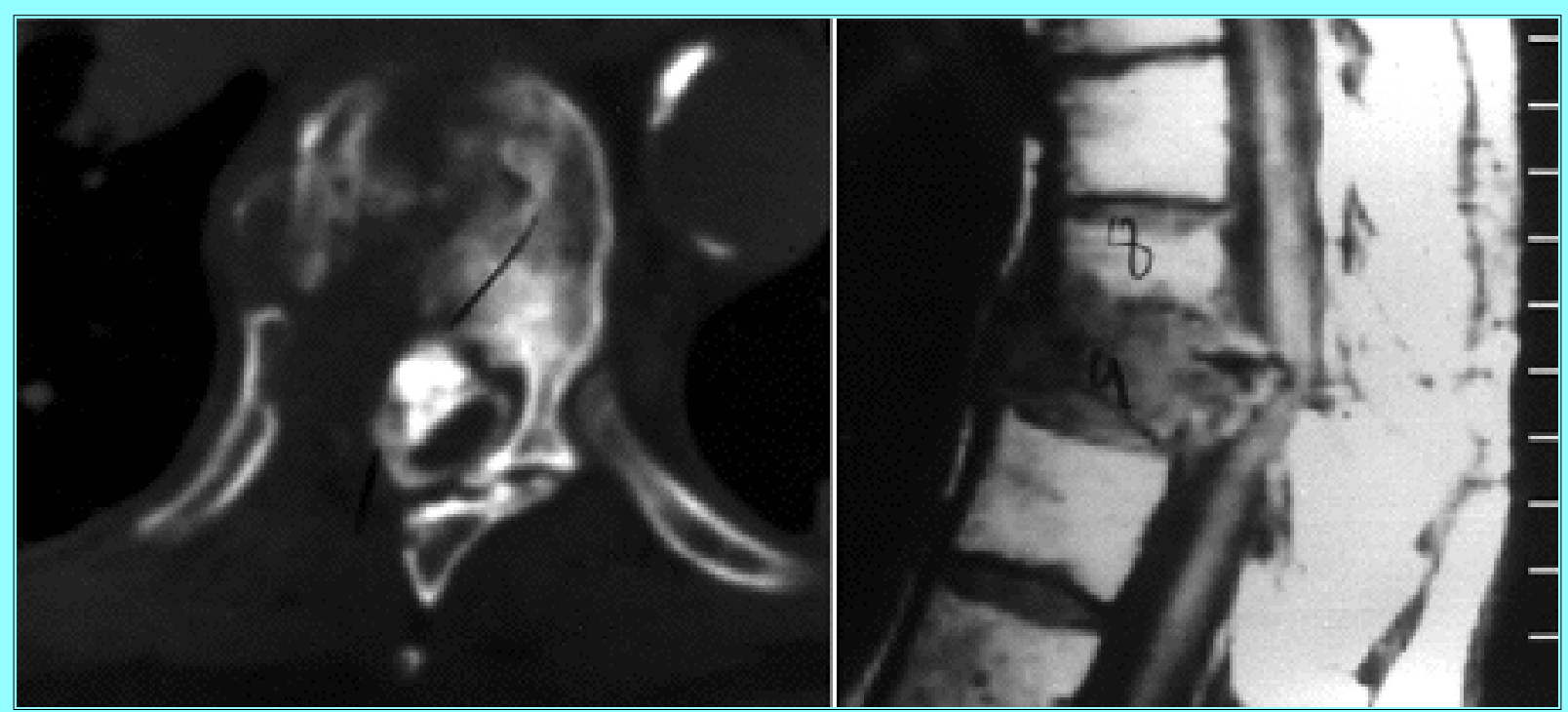

Fig. 1. Imaging studies. Left: Postmyelography axial CT scan obtained in a patient who had undergone a prior transpedicular approach that failed to remove the original transdural calcified thoracic disc herniation at T8-9. The patient's initial operation was aborted when the somatosensory evoked potentials dropped out during an attempt to manipulate the disc fragment. Only a small cavity was created in the T-9 vertebral body. The base of the calcified disc was partially disconnected from the vertebral body. The spinal cord was compressed, distorted, and displaced by the residual disc. Right: Sagittal MR image revealing spinal cord compression caused by the residual disc. This patient deteriorated neurologically and developed progressive myelopathy secondary to the residual compression.

There were often multiple reasons for reoperation: misidentification of the level of the symptomatic disc at initial operation (five cases), abandoning the operative procedure because of intraoperative neurological injury (three cases), inadequate visualization of the lesion (eight cases), intraoperative migration of a soft disc fragment within the spinal canal (one case), and transdural extension of the disc (three cases). 
The time interval between the initial surgery and reoperation was less than 24 hours in three patients. Of the patients who underwent reoperation during the acute phase, mislocalized levels that were recognized on postoperative radiographs prompted these reoperations in two patients. In one patient migration of a soft disc herniation within the spinal canal, cephalad to the original site, was demonstrated. The residual disc in this patient caused neurological deterioration, and reoperation was performed urgently. The time interval between the initial surgery and reoperation was longer than 1 month in 12 patients. The average interval was 150 days (range 1-48 months) in this group.

In all 15 cases of reoperation, an anterolateral approach was performed using video-assisted thoracoscopy (14 patients) or thoracotomy (one patient).

Eleven reoperative proceedures were performed ipsilateral and four contralateral to the site of the initial surgery. Even in the one patient who had undergone a prior thoracotomy, lung adhesions were mild or moderate and could be detached endoscopically to allow access for reoperation using thoracoscopy.

The procedure for anterior thoracic discectomy has been described previously.[7,22,25] Briefly, the rib head and pedicle were removed to expose the dura. A cavity was created in the dorsal disc space and vertebral body to provide enough working space to move the disc material away from the spinal canal, thereby facilitating the entry of any tools into the epidural space. For the removal of large ossified or intradural discs, a corpectomy was required to expose fully the spinal cord (Figs. 2 \& 3). 


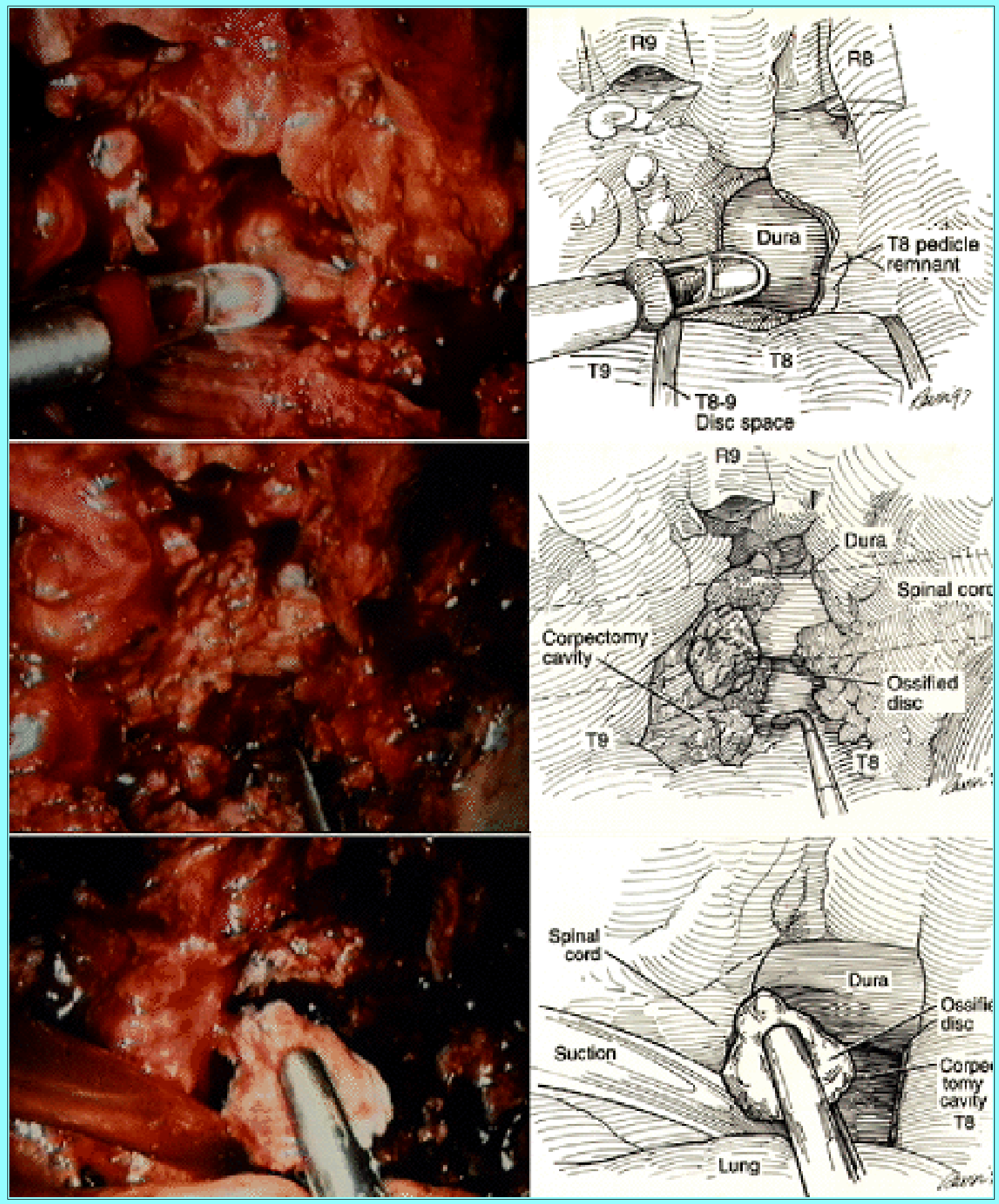

Fig. 2. Intraoperative photographs obtained in the patient shown in Fig. 1, and corresponding illustration. Intraoperative photograph 1 (upper left) and corresponding illustration depicting the right-sided thoracoscopic approach used to resect this calcified disc using a partial corpectomy (upper right). The rib heads and pedicles were removed to expose the lateral surface of the dura. Intraoperative photograph (center left) and corresponding illustration depicting the corpectomy cavity created in the vertebral body to expose the entire ventral and lateral surfaces of the dura at the site where the spinal cord was compressed (center right). This large cavity created a working space to allow the disc to be removed from the surface of the spinal cord by using endoscopic microdissection tools. Intraoperative photograph (lower left) and corresponding illustration depicting the calcified 
disc fragment separated from the dura and removed with a disc rongeur (lower right). All operative illustrations are used with permission from Barrow Neurological Institute.

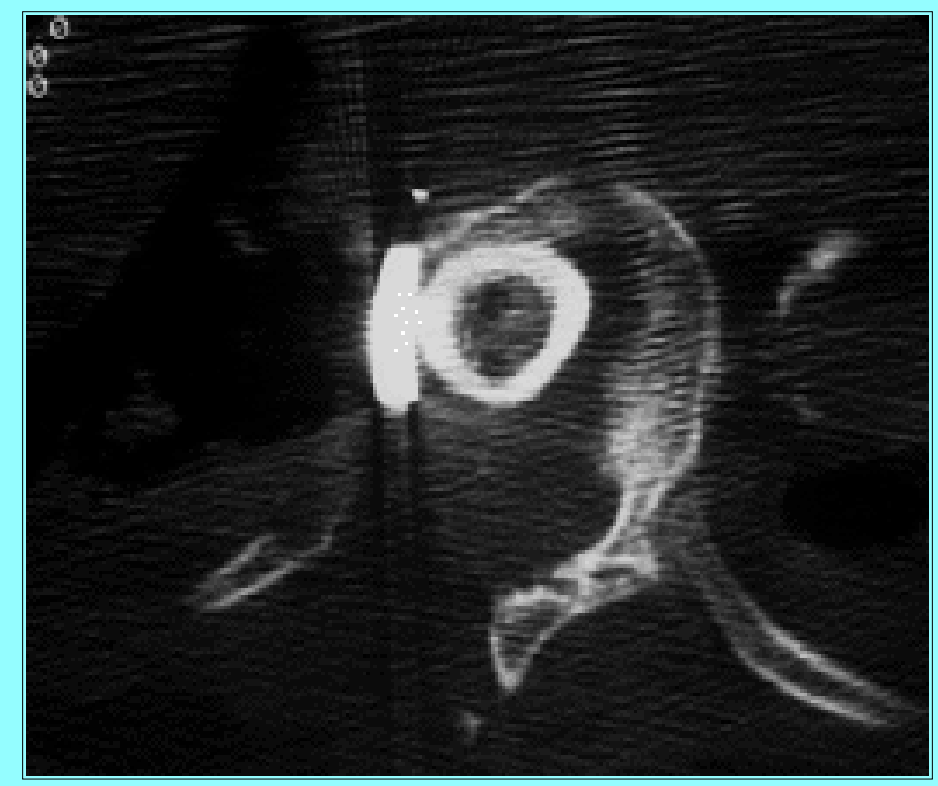

Fig. 3. Postoperative CT scan revealing a complete decompression. The corpectomy was reconstructed using a humerus allograft and a screw plate.

Of the 15 patients, 10 also required adjunctive spinal reconstruction and internal fixation procedures to restore or maintain spinal stability. Stabilization was considered when a corpectomy was performed or when extensive bone was resected circumferentially (that is, when both anterior and posterolateral approaches had been performed) at the same level during the multiple operative procedures. The spinal reconstruction and fixation procedures consisted of eight anterior interbody bone grafts: in seven patients spinal instrumentation was placed, three patients had anterior screw plates, and four patients had posterior spinal fixations (Table 2).

\begin{tabular}{|c|c|}
\hline $\begin{array}{c}\text { TABLE } 2 \\
\text { SUMMAV OF SPINAL RECONSTRUCTON AND INSTRUMENTAT } \\
\text { PATIENTS WITH RESIDUAL THORACIC DISCS }\end{array}$ & $D \| \mathbb{N}$ \\
\hline $\begin{array}{l}\text { anterior interbody stut grafts wh instrumentation } \\
\text { anterior interbody stut grafts w/anterior screw-plate fixation } \\
\text { posterior spinal instrumentation \& posterior fusion } \\
\text { Luque rods w/Drummond wires \& cables } \\
\text { uni versal hook-rod instrumentation }\end{array}$ & $\begin{array}{l}3 \\
3 \\
4 \\
(2) \\
(2)\end{array}$ \\
\hline $\begin{array}{l}\text { xAll } 7 \text { patients who recei ied spinal instrumentation had } \\
\text { costotransiersectomy with laminectom y and facetectomy dur } \\
\text { operati ie approach. The reoperations were all performed usin } \\
\text { proach in which corpectomies were performed to excise the re } \\
\text { circum ferential resection of bone anteriorly and posteriorlyres } \\
\text { ty and required internal fixation. }\end{array}$ & $\begin{array}{l}\text { one prior } \\
\text { r original } \\
\text { terior ap- } \\
\text { iscs. The } \\
\text { instatili- }\end{array}$ \\
\hline
\end{tabular}

In the three patients with intradural disc herniations, lumbar drains and/or lumboperitoneal shunts were placed to prevent or treat a cerebrospinal fluid (CSF) subarachnoid-pleural fistula. The dura was sutured closed or covered with fibrin glue and muscle or fascia after transdural disc material was removed.

Fourteen of the discs were removed completely during a single reoperative procedure. One patient who harbored a large, residual calcified intradural disc from a prior costotransversectomy required two staged 
reoperations. At the first, a portion of the intradural stalk of the disc was obscured by the edges of the dura enfolded around the intradural disc extension. This residual component was recognized on the postoperative CT scan, and the residual disc was removed in a second reoperative procedure the next day without sequelae (Figs. 4-6).
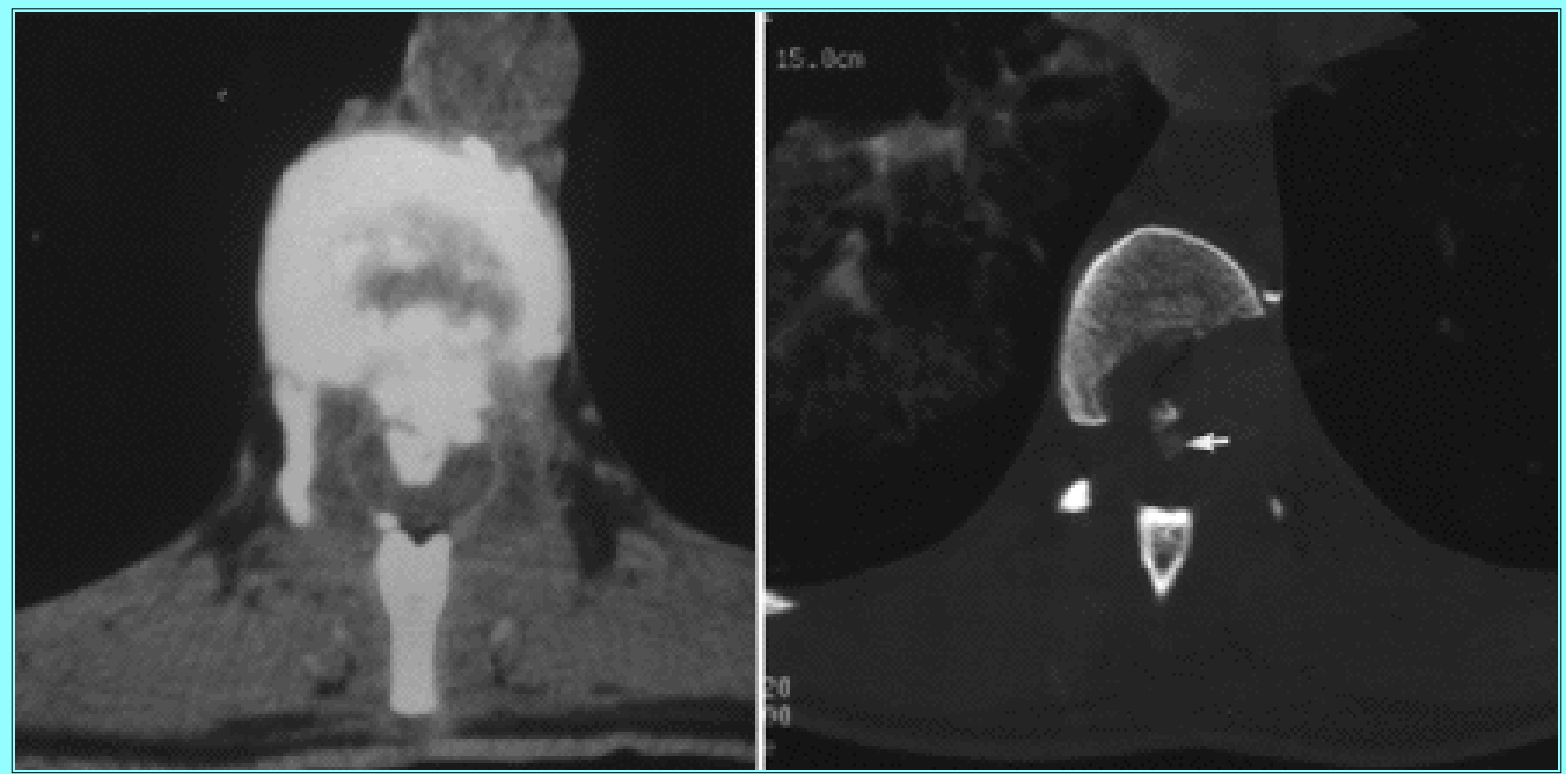

Fig. 4. A CT study in a patient who had undergone a prior bilateral costotransversectomy and transpedicular approach that had failed to remove this massive, lobular, ossified, transdural disc herniation. The patient sustained an intraoperative spinal cord injury during the initial surgery. Left: Postoperative axial CT scan revealing the residual calcified disc compressing and distorting the spinal cord. The left pedicle, part of the right pedicle, and the ribs were removed at this level. A left-sided thoracoscopic approach was then used to perform a corpectomy to resect the disc herniation. Right: Postoperative CT scan after the second surgery revealing a residual calcified disc fragment (arrow) compressing the spinal cord.
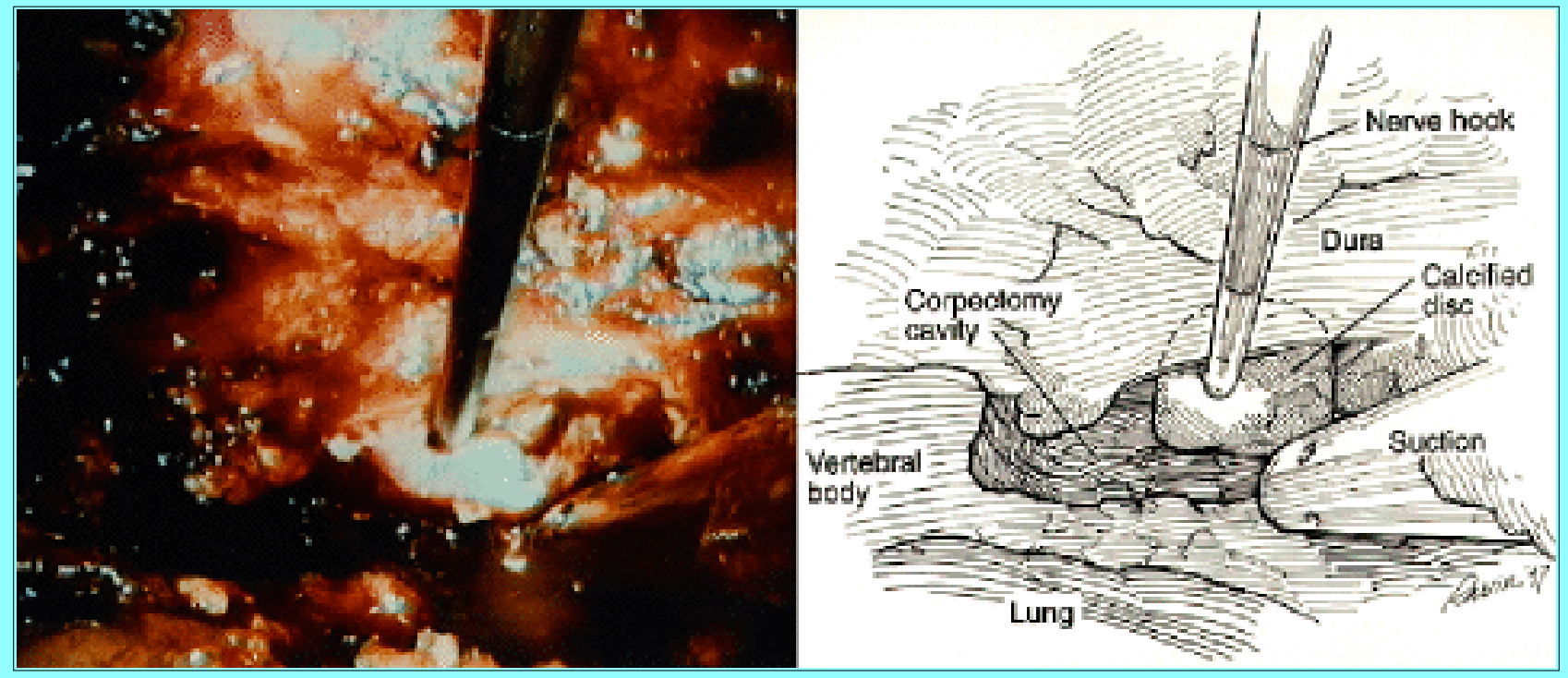

Fig. 5. Intraoperative thoracoscopic photograph (left) and corresponding illustration (right) obtained in the same patient in Fig. 4 revealing the exposure and removal of the residual intradural disc fragment. The disc was removed completely, the dura was closed using fibrin glue, and a prophylactic lumbar drain was placed to reduce the hydrostatic pressure of the 
CSF. Postoperatively, the patient gradually and progressively recovered neurological function and was normal at a 2-year follow-up evaluation. Illustration provided with permission from Barrow Neurological Institute.

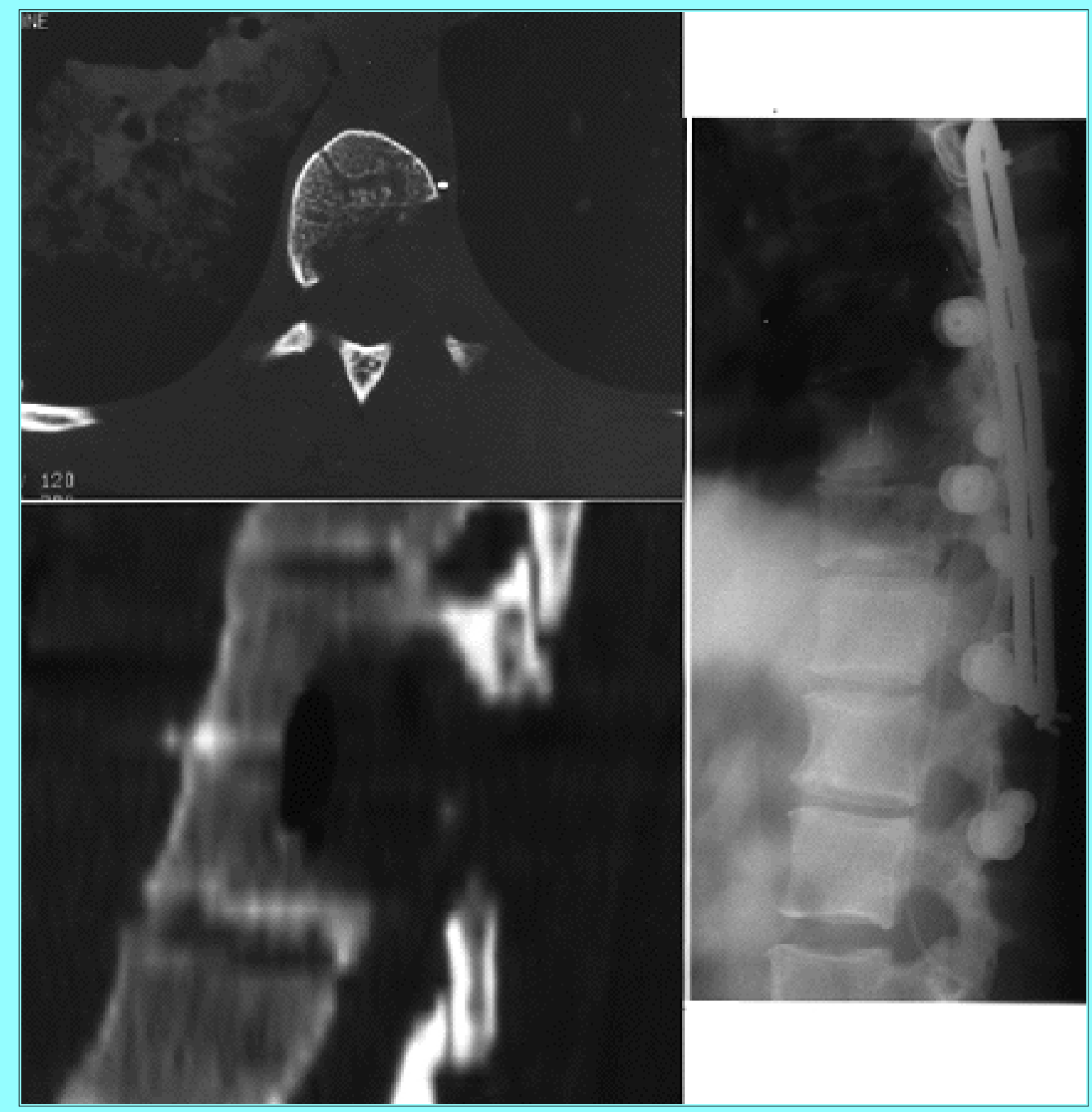

Fig. 6. Postoperative axial (upper) and sagittal (lower) CT scans obtained the same patient in Figs. 4 and 5 revealing the corpectomy cavity and complete resection of the disc. Right: Postoperative lateral thoracic radiograph revealing that the posterior fixation and fusion of the thoracic spine have restored spinal stability.

Complications of the reoperation included three cases of atelectasis, two cases of transient intercostal neuralgia, and one case of CSF collection within the pleural space. In one patient with severe osteoporosis removal of a loose anterior fixation screw was also required, but there were no sequelae. None of the reoperations caused new neurological deficits. With treatment, all cases of atelectasis, CSF leak, and intercostal neuralgia were completely resolved without sequelae.

Eleven of the 15 patients underwent followup of at least 2 years. At follow-up evaluation (mean 26 months, range 10-46 months), clinical improvement of neurological function was demonstrated in 10 of the 13 patients with myelopathy; in three patients myelopathy stabilized (Table 3). The isolated radicular 
pain improved in both patients with radiculopathy. Three of the 15 patients experienced mild or moderate chronic local spine pain but no radicular symptoms or disabling pain. The pain was attributed to local irritation of the tissue around the patient's posterior instrumentation in all three cases. No patients developed nonunion or delayed spinal instability. Postoperative imaging studies revealed in all patients that a complete decompression of the spinal cord and nerve roots was achieved. Radiographic evaluation of patients who underwent a fusion procedure in which instrumentation was used (all had a minimum of 2 years of follow up) revealed no cases of nonunion or instrumentation failure.

\begin{tabular}{|cccccc|}
\hline \multicolumn{5}{c|}{ TABLE 3 } \\
NEUROLOGICAL OUTCOME IN 15 PATIENTS \\
WHOSE RESIDUAL DISKS WERE REMOVED VIA \\
THE AN TERIOR APPROACH \\
\hline \hline $\begin{array}{c}\text { Preop } \\
\text { Franke } \\
\text { Grade }\end{array}$ & \multicolumn{5}{c}{ Postop Frankel Grade } \\
\cline { 2 - 6 } & A & B & C & D & E \\
\hline A & & & & & \\
B & 1 & & & \\
C & & & 1 & 3 & \\
D & & & & 1 & 7 \\
E & & & & & \\
\hline
\end{tabular}

\section{DISCUSSION}

The resection of thoracic disc herniations can present formidable technical difficulties, especially if the discs are calcified, ossified, large, centrally located, broad based, or extend transdurally. Residual disc herniations that cause neurological symptoms secondary to spinal cord and nerve root compression tend to cause progressive deficits and should be considered for reoperation.

The two most common reasons for failure to remove a thoracic disc were mislocalization of the disc level and inadequate visualization of the diseased entity. The latter principally reflects choosing the wrong route of operative exposure. Although it is a problem inherent to operating in the thoracic spine, mislocalization can usually be avoided by carefully counting the number of ribs on preoperative chest radiographs and by using intraoperative anteroposterior fluoroscopy and radiographs to identify the disc space. If an anterior approach is used, the correct location can be confirmed by internally counting the rib heads. Other landmarks such as osteophytes can also be used to aid in localization.

Inadequate visualization of the pathology can be prevented by selecting an approach that provides a full view of the ventral spinal cord and herniated disc material and by removing enough of the vertebral body to ensure full visualization of the spinal cord. A corpectomy is often necessarily performed to identify normal dura above and below a large disc. The posterior longitudinal ligament should be removed to view the dura adequately.

\section{Posterolateral Approaches}

Posterior or posterolateral approaches provide an oblique view of the spinal canal (Fig. 7). This view does not permit visualization of the ventral surface of the spinal cord. Even if bilateral transpedicular or 
costotransversectomy approaches are used, the surgeon cannot visualize the ventral dural surface or the interface of the ventral midline dura and the pathology. We believe that this inadequate visualization and exposure (by failing to make a large working space in the vertebral body) contributed to the spinal cord injuries that patients sustained during the original operations in which the posterolateral approaches were performed. Transpedicular or posterolateral approaches are indicated for treatment of small, laterally positioned thoracic disc herniations. However, the findings in our patients suggest that posterior or posterolateral approaches are contraindicated in patients with large, central, densely calcified, or transdural thoracic discs because they are associated with a risk of neurological deficits, spinal cord injury, and incomplete disc removal.

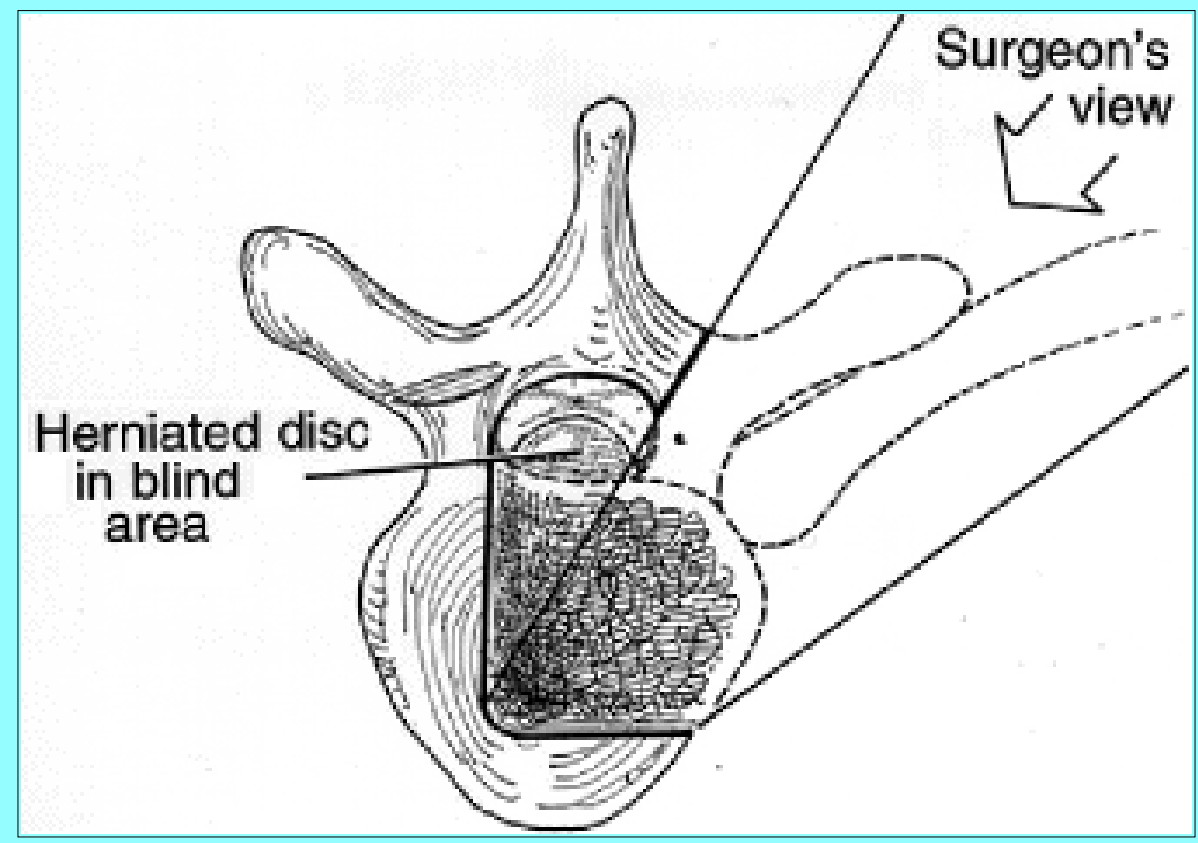

Fig. 7. Illustration revealing how posterolateral approaches to the thoracic spine do not permit the surgeon to visualize the ventral surface of the dura and spinal cord. Only the posterior and lateral surfaces of the dura are visible. The lateral third to half of the dorsal disc space and vertebral body can be viewed with this approach. The surgeon cannot see the ventral midline surface of the dura using this exposure. Illustration used with permission from Barrow Neurological Institute.

\section{Anterior Approaches}

An anterolateral approach, in which thoracotomy is performed or a thoracoscope is used, provides direct full visualization of the entire ventral surface of the dura and the ventral spinal cord (Fig. 8). The anterior approach is indicated for the safe microsurgical removal of large, calcified, broad-based, midline, and transdural discs. A thoracotomy, which is an excellent alternative, has been the traditional anterior approach to the thoracic spine, is associated with excellent results for treatment of herniated thoracic discs, and may be the preferred approach for transdural discs to allow water-tight suturing of the dura. Thoracotomy is associated with a moderate incidence of postoperative pulmonary and shoulder dysfunction as well as postthoracotomy pain syndrome.[5,8,9,12,23] In our experience, with proper training, thoracoscopy has become the procedure of choice when an anterior approach is required to resect a herniated thoracic disc. Video-assisted thoracoscopy has several theoretical advantages over thoracotomy. It appears to be associated with less postoperative pain, fewer pulmonary complications, and faster recoveries; however, definitive data are required to assess these issues 


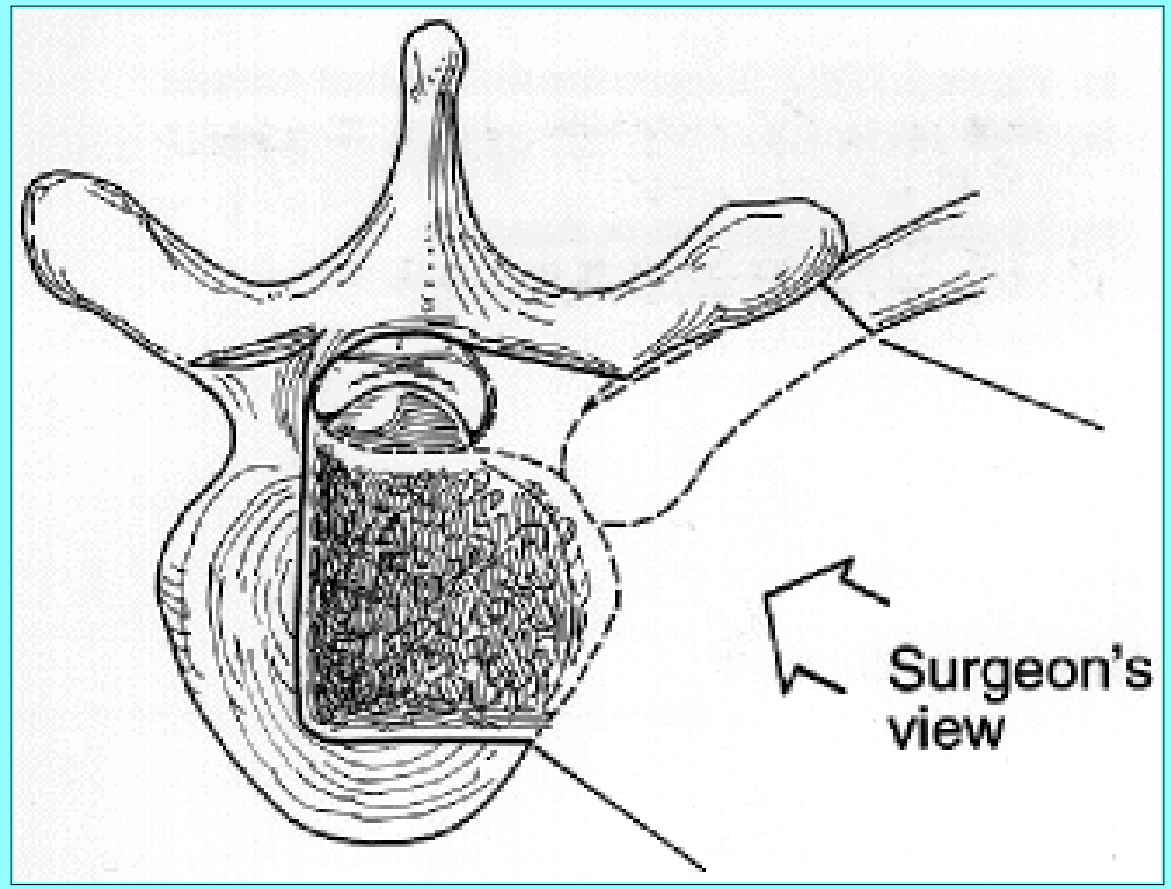

Fig. 8. Illustration revealing how anterior approaches to the thoracic spine provide a panoramic view of the entire ventral surface of the dura and the spinal cord. Both thoracotomy and thoracoscopy provide this perspective, which is needed to permit safe microsurgical dissection of large, calcified, midline, broad-based, and intradural thoracic disc herniations. Used with permission from Barrow Neurological Institute.

\section{Thoracoscopy-Guided Surgery}

In experienced hands, video-assisted thoracoscopic surgery provides an excellent technique for both primary operations and reoperations in the treatment of herniated thoracic discs. In addition to being associated with a lower morbidity rate and a faster recovery time than thoracotomy, thoracoscopy-guided surgery provides direct access and visualization of the entire ventral surface of the spinal cord, which facilitates the procedure. $[6,10,15,22-25]$ If the surgeon is inexperienced or lacks the training required to perform thoracoscopic discectomy safely, thoracotomy is recommended when a ventral approach is required for reoperation.

Thoracoscopy can be used successfully for the reoperation of thoracic discs despite the presence of pleural adhesions, scar tissue at the operative site, and distorted spinal anatomy. Thoracoscopy requires that pleural adhesions, if present, be detached to obtain spinal exposure. This option may not always be feasible if the patient has undergone a prior thoracotomy and has dense lung adhesions. Prior pleurodesis or an empyema or hemothorax can also preclude use of thoracoscopy.

To perform thoracoscopic discectomy in patients definitely requires extensive training, laboratory practice, new skill development, and mastering of the surgical techniques. Realization of these skills will enhance patient outcome and minimize complications associated with this surgical technique.

\section{Intradural Discs}

An open thoracotomy is currently the preferred procedure for microsurgical resection of intradural discs. If the discs extend transdurally, water-tight dural closure can be best achieved using direct suturing rather 
than endoscopically assisted suturing. Endoscopically, dural closure can be achieved using fibrin glue and covering the dura with a muscle or fascial graft. Lumbar drainage and avoiding negative pressure on the chest tube (that is, placing the chest tube to water seal) are strategies to avoid a CSF-pleural fistula. The lumbar drain should be kept in place for at least several days after the chest tubes are removed.

\section{Role of Fusion}

Most routine thoracic disc herniations do not require instrumentation and fusion because the facets, rib cage, and remaining thoracic spine adequately stabilize the spine. However, if a corpectomy is required to resect a large thoracic disc, or if in performing multiple anterior and posterior approaches to resect a residual disc the spine has become destabilized, then internal fixation and fusion are required. In more than half of the patients who underwent reoperations for thoracic discs spinal fusion was required. In all patients posterior approaches had been used (costotransversectomy with laminectomy and facetectomy) and anterior-approach reoperations were performed with partial or subtotal corpectomies; by using the combination of procedures extensive bone was resected circumferentially across the vertebral column. In contrast, fusion is rarely required after a first operation for resection of a herniated thoracic disc.

\section{CONCLUSIONS}

Based on our experience in performing reoperations for the treatment of herniated thoracic discs, several conclusions can be drawn. 1) Thoracic discs can be difficult to resect if the disc herniations are calcified, large, broad based, centrally located, or intradural. These lesions require a ventral operative approach to obtain adequate visualization of the dura to resect the discs completely. 2) Residual disc herniations that cause spinal cord or nerve root compression tend to cause progressive neurological deficits and should be treated with reoperation. 3) Posterior and posterolateral approaches to the thoracic spine provide an oblique view of the ventral spinal cord, a view that does not permit visualization of the ventral spinal cord. These approaches are best suited for small, soft, or calcified disc herniations that are laterally positioned within the spinal canal. The posterolateral approaches are contraindicated for large, central, densely calcified, or transdural discs because they carry a high risk of neurological deficits, spinal cord injury, and incomplete disc removal. 4) Thoracotomy and thoracoscopy are both excellent alternatives for ventral access to the thoracic spine. 5) Video-assisted thoracoscopic surgery provides an excellent technique for reoperation that can be used successfully despite the presence of scar tissue at the operative site, distorted spinal anatomy, and focal pleural adhesions. In experienced hands, the use of thoracoscopy minimizes morbidity and facilitates recovery, while providing direct access and visualization of the entire ventral surface of the spinal cord.

\section{References}

1. Arseni C, Nash F: Thoracic intervertebral disc protrusion. A clinical study. J Neurosurg 17:418-430, 1960

2. Benjamin V: Diagnosis and management of thoracic disc disease. Clin Neurosurg 30:577-605, 1983

3. Bohlman HH, Zdeblick TA: Anterior excision of herniated thoracic discs. J Bone Joint Surg (Am) 70:1038-1047, 1988

4. Carson J, Gumpert J, Jefferson A: Diagnosis and treatment of thoracic intervertebral disc protrusions. J Neurol Neurosurg Psychiatry 34:68-77, 1971 
5. Dajczman E, Gordon A, Kreisman H, et al: Long-term postthoracotomy pain. Chest 99:270-274, 1991

6. Dickman CA, Karahalios DG: Thoracoscopic spinal surgery. Clin Neurosurg 43:392-422, 1996

7. Dickman CA, Mican C: Thoracoscopic approaches for the treatment of anterior thoracic spinal pathology. BNI Q 12 (1):4-19, 1996

8. Faciszewski T, Winter RB, Lonstein JE, et al: The surgical and medical perioperative complications of anterior spinal fusion surgery in the thoracic and lumbar spine in adults. A review of 1223 procedures. Spine 20:1592-1599, 1995

9. Ferson PF, Landreneau RJ, Dowling RD, et al: Comparison of open versus thoracoscopic lung biopsy for diffuse infiltrative pulmonary disease. J Thorac Cardiovasc Surg 106:194-199, 1993

10. Horowitz MB, Moossy JJ, Julian T, et al: Thoracic discectomy using video assisted thoracoscopy. Spine 19:1082-1086, 1994

11. Hulme A: The surgical approach to thoracic intervertebral disc protrusions. J Neurol Neurosurg Psychiatry 23:133-137, 1960

12. Landreneau RJ, Hazelrigg SR, Mack MJ, et al: Postoperative pain-related morbidity: video-assisted thoracic surgery versus thoracotomy. Ann Thorac Surg 56:1285-1289, 1993

13. Love JG, Schorn VG: Thoracic-disk protrusions. JAMA 191:91, 1965

14. Maiman DJ, Larson SJ, Luck E, et al: Lateral extracavitary approach to the spine for thoracic disc herniation: report of 23 cases. Neurosurgery 14:178-182, 1984

15. McAfee PC, Regan JR, Zdeblick T, et al: The incidence of complications in endoscopic anterior thoracolumbar spinal reconstructive surgery. A prospective multicenter study comprising the first 100 consecutive cases. Spine 20:1624-1632, 1995

16. Naunheim KS, Barnett MG, Crandall DG, et al: Anterior exposure of the thoracic spine. Ann Thorac Surg 57:1436-1439, 1994

17. Otani K, Nakai S, Fujimura Y, et al: Surgical treatment of thoracic disc herniation using the anterior approach. J Bone Joint Surg (Br) 64:340-343, 1982

18. Otani K, Yoshida M, Fujii E, et al: Thoracic disc herniation. Surgical treatment in 23 patients. Spine 13:1262-1267, 1988

19. Patterson RH Jr, Arbit E: A surgical approach through the pedicle to protruded thoracic discs. J Neurosurg 48:768-772, 1978

20. Perot PL Jr, Munro DD: Transthoracic removal of midline thoracic disc protrusions causing spinal cord compression. J Neurosurg 31:452-458, 1969

21. Ransohoff J, Spencer F, Siew F, et al: Transthoracic removal of thoracic disc. Report of three cases. J Neurosurg 31:459-461, 1969

22. Regan JJ, Mack MJ, Picetti GD III: A technical report on video-assisted thoracoscopy in thoracic spinal surgery. Preliminary description. Spine 20:831-837, 1995 
23. Regan JJ, Mack MJ, Picetti GD III, et al: A comparison of video-assisted thoracoscopic surgery (VATS) with open thoracotomy in thoracic spinal surgery. Today Ther Trends 11:203-218, 1994

24. Rosenthal D, Lorenz R: The use of the microsurgical endoscopic technique for treating affections of the dorsal spine: indications and early results. J Neurosurg 82:342A, 1995 (Abstract)

25. Rosenthal D, Rosenthal R, de Simone A: Removal of a protruded thoracic disc using microsurgical endoscopy. A new technique. Spine 19:1087-1091, 1994

26. Sekhar LN, Jannetta PJ: Thoracic disc herniation: operative approaches and results. Neurosurgery 12:303-305, 1983

27. Stillerman CB, Chen TC, Day JD, et al: The transfacet pedicle-sparing approach for thoracic disc removal: cadaveric morphometric analysis and preliminary clinical experience. J Neurosurg 83:971-976, 1995

28. Stillerman CB, Weiss MH: Surgical management of thoracic disk herniation and spondylosis, in Menezes AH, Sonntag VKH (eds): Principles of Spinal Surgery. New York: McGraw-Hill, 1996, pp 581-601

Manuscript received October 16, 1998.

Accepted in final form April 1, 1999.

Address reprint requests to: Curtis A. Dickman, M.D., c/o Neuroscience Publications, Barrow Neurological Institute, 350 West Thomas Road, Phoenix, Arizona 85013-4496 e-mail: neuropub@mha.chw.edu. 